Few-Body Systems Suppl. 0, 1-2 (2018)

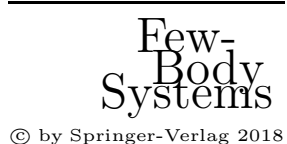

() by Springer-Verla
Printed in Austria

\title{
Three quark clusters in hot and dense matter
}

\author{
S. Mattiello ${ }^{a *}$, M. Beyer $^{a}$, T. Frederico ${ }^{b}$, H. J. Weber ${ }^{c}$ \\ ${ }^{a}$ Fachbereich Physik, Universität Rostock, D-18051 Rostock, Germany \\ ${ }^{b}$ Dep. de Física, ITA, CTA, 12.228-900 São José dos Campos, Brazil \\ ${ }^{c}$ Dept. of Physics, University of Virginia, Charlottesville, VA 22904, U.S.A.
}

The phase diagram of quantum chromodynamics (QCD) shows a rich structure as lattice simulations and model calculations indicate [1]. One very interesting aspect is the transition from quarks to nucleons as relevant degrees of freedom (Mott transition [2, 34). This is related to the transition between the hadronic matter and the quark-gluon plasma phase. Another particularly interesting possibility is quark pairing that leads to the color superconductivity analogous to Cooper pairing. An aspect that has hardly been investigated in this context is the appearance of three-quark correlations that, in the vicinity of the phase transition, should play an important role and might as well influence quark pairing. Here we address the issue of three-quark clusters in a medium of finite temperatures and densities. This necessarily involves relativity as well as effects of the medium. To implement relativity we use the light front approach and utilize a Green function formalism. We consider first the isolated case and then medium effects. Using a zero-range interaction, the two-body propagator is $t=\left(i \lambda^{-1}-B\left(M_{2}\right)\right)^{-1}$, where $B\left(M_{2}\right)$ denotes the loop

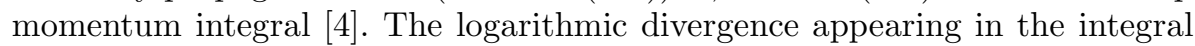
can be eliminated by introducing an invariant cut-off $\Lambda$. The requirement is that $\Lambda$ is smaller than the intermediate mass of the virtual two-quark subsystem, i.e. $M_{20}^{2}<\Lambda^{2}$ and hence $t \rightarrow t_{\Lambda}$. We introduce a similar regularisation for the three-body equation $\left(M_{30}^{2}<\Lambda^{2}\right)$ that leads to an equation for the vertex function $\Gamma_{\Lambda}$ that depends parametrically on the cut-off $\Lambda$,

$$
\begin{gathered}
\Gamma_{\Lambda}\left(M_{3} ; y, \boldsymbol{q}_{\perp}\right)=\frac{i}{(2 \pi)^{3}} t_{\Lambda}\left(M_{2}\right) \int_{x_{\min }}^{1-y} \frac{d x}{x(1-y-x)} \\
\int d^{2} k_{\perp} \frac{\theta\left(M_{03}^{2}-\Lambda^{2}\right)}{M_{3}^{2}-M_{03}^{2}} \Gamma_{\Lambda}\left(M_{3} ; x, \boldsymbol{k}_{\perp}\right) .
\end{gathered}
$$

In Fig. 1 1 we show the mass of the three-quark cluster as function of the diquark mass for different cut-offs in units of the quark mass. Note that $M_{3} \rightarrow 0$ for

*E-mail address: stefano.mattiello@physik.uni-rostock.de 


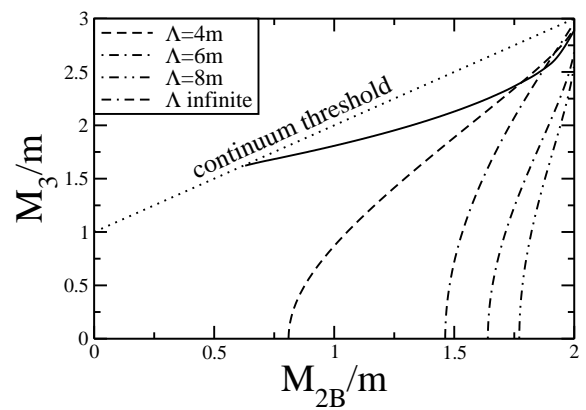

Figure 1. $M_{3}$ as function of the mass of the "diquark" $M_{2 B}$.

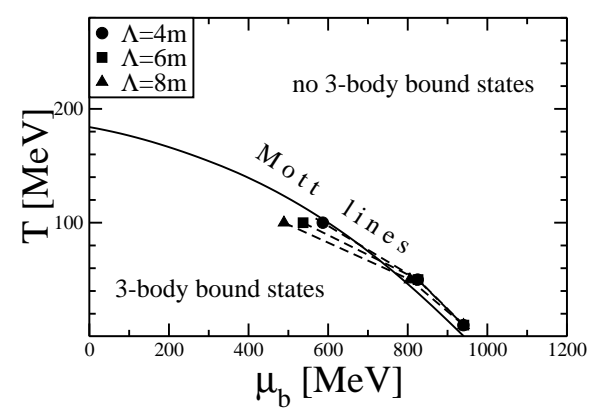

Figure 2. Phase-diagram of QCD. See text for explanation.

finite diquark masses which is the relativistic analog of the Thomas collapse [5]. The results using the previous regularisation [4] are shown as solid line.

To investigate medium effects we fit the parameters $(\Lambda, \lambda)$ to reproduce the proton mass. The three-body equation systematically includes the dominant medium effects due to Pauli blocking and self energy corrections, $m \rightarrow m(T, \mu)$. For a given temperature and a given choice of the fit parameters, the threequark system becomes more weakly bound, as the baryonic chemical potential $\mu_{\mathrm{b}}$ increases. The continuum is reached for binding energy $B_{3}=0$ (Mott transition). The Mott lines, i.e. the values of $T$ and $\mu_{\mathrm{b}}$ where this transition occurs are given in Fig. 2. We show our previous result (solid line) along with the ones using the invariant cut-off. From other models investigating $q \bar{q}$ states the confinement-deconfinement phase transition has been estimated as well. We find a similar qualitative behavior for the three-body Mott lines. A closer comparison indicates differences at larger densities that are currently investigated. The next aim is to investigate the phase transition between the color superconducting phase and the quark matter considering the influence of threequark-correlations.

\section{References}

1. Rajagopal K., Wilczek F.: Handbook of QCD, M Shifman ed. (World Scientific 2001)

2. Mattiello S., Beyer M., Frederico T., Weber H. J.: Few Body Syst., 31 (2002) 159

3. Beyer M., Mattiello S., Frederico T., Weber H. J.: Phys. Lett. B 521, 33 (2001)

4. Frederico T.: Phys. Lett. B 282, 409 (1992)

5. Thomas L. H.: Phys. Rev. 47, 903 (1935) 